\section{Pediatric Neurosurgery}

Berger, D. 426

Bonow, R.H. 381

Browd, S.R. 381

Castaneyra-Ruiz, L. 426

Dewan, M.C. 401

George, T.M. 361

Guerra, M.M. 446

Hale, A.T. 361

Han, R.H. 426

Hanak, B.W. 381

Harris, C.A. 381
Klarica, M. 417

Limbrick Jr, D.D. 426

Mangano, F.T. 436

McAllister, J.P. 426

Morales, D.M. 426

Naftel, R.P. 401

Orešković, D. 417

Patel, S.K. 436
Radoš, M. 417

Rodríguez, E.M. 446

Smith, A.J. 409

Tomei, K.L. 369

Tomycz, L.D. 361

Tradtrantip, L. 409

Verkman, A.S. 409, 409

Yao, X. 409

Yuan, W. 436

\title{
Subject Index Vol. 52, No. 6, 2017
}

Aquaporins 409

Benign external hydrocephalus 436

Biomarker 426

Bulat-Klarica-Orešković hypothesis 417

Catheter obstruction 381

Cerebrospinal fluid 409, 417, 426

- - pathophysiology 417

- - pressure 417

Choroid plexus 409, 417

- - cauterization 401

Congenital hydrocephalus 426, 446

Diffusion tensor imaging 436

Endoscopic third ventriculostomy 401

Ependymogenesis 446
Fractional anisotropy 436

Hydrocephalus 369, 381, 401, 409, 417, 426

Intracranial pressure 417

Intraventricular hemorrhage 426

Magnetic resonance elastography 436

- - imaging 436

Mean diffusivity 436

Neural stem cell grafting 446

- _ - pathology 446

Neurogenesis 446

New hydrocephalus definition 417

Pediatric hydrocephalus 436

Posthemorrhagic hydrocephalus 426

Programmable valve 369
Shunt 369

- failure 381

- technology 369

Structural network connectivity 436

Subventricular zone 446

Synthetic MRI 436

Ventricular zone 446

- - disruption 446

Ventriculoatrial shunt 381

Ventriculoperitoneal shunt 381

Ventriculopleural shunt 381

Water channels 409

Weed-Dandy-Cushing hypothesis 417

White matter damage 436 